Cytologia Focus:

\title{
Stem Cell Maintenance in the Shoot Apical Meristems and during Axillary Meristem Development
}

\author{
Hiro-Yuki Hirano ${ }^{1 *}$ and Wakana Tanaka ${ }^{1,2}$ \\ ${ }^{1}$ School of Science, The University of Tokyo, 7-3-1 Hongo, Bunkyo-ku, Tokyo 113-8654, Japan \\ ${ }^{2}$ Graduate School of Integrated Sciences for Life, Hiroshima University, 1-4-4 Kagamiyama, Higashi-Hiroshima 739-8528, Japan
}

Received December 26, 2019; accepted January 22, 2020

\begin{abstract}
Summary In plants, the shoot apical meristem (SAM), which is formed during embryogenesis, plays a crucial role in postembryonic development of the above-ground shoots. The SAM harbors pluripotent stem cells, which provide daughter cells to differentiate lateral organs and stems. As the plant grows, the primary shoot generates the axillary meristems, which are responsible for secondary shoot development. During the development of axillary meristem, stem cells must be established and maintained to build up the axillary meristems. While the mechanism underlying stem cell maintenance in the SAM, including the WUSCHEL-CLAVATA negative feedback loop, has been well elucidated, studies on the regulation of stem cell maintenance during axillary meristem development have only just begun. In this minireview, we describe the genetic mechanisms underlying stem cell maintenance in both the established SAM and the developing axillary meristem in two model plants, Arabidopsis thaliana and Oryza sativa (rice). We also discuss the function of genes that are specific or shared between the established and developing meristems or between Arabidopsis and rice.
\end{abstract}

Keywords Axillary meristem, CLAVATA, Rice, Shoot apical meristem, Stem cell, WUSCHEL.

Plant architecture is determined by various morphological traits, such as the number and pattern of branches, size and shape of leaves, morphology of flowers, and type of inflorescence (Yamaguchi et al. 2010, Nakayama et al. 2012, Dhaliwal et al. 2018, Kumar et al. 2018, Nasirvand et al. 2018, Shiogai et al. 2018, reviewed in Bell 2008, Soltis and Soltis 2014, Tsukaya 2018). The branches are formed from the axillary buds, which develop in the axil of the leaves. Branch formation comprises two developmental stages: (i) initiation and establishment of the axillary bud in the leaf axil, and (ii) outgrowth of the axillary shoot from the bud. A large number of genes responsible for transcription and signaling, in addition to phytohormones such as auxin and strigolactone, regulate these two developmental events (reviewed in Domagalska and Leyser 2011, Wang et al. 2016, 2018, Barbier et al. 2019). Accumulating studies on hormone interactions and genetic networks have provided deep understanding of the mechanism that regulates axillary bud growth. By contrast, the molecular genetic mechanisms underlying the initiation and establishment of the axillary bud remain to be elucidated in detail.

The axillary bud comprises the axillary meristem and leaf primordia. The axillary meristem contains pluripo-

\footnotetext{
* Corresponding author, e-mail: hyhirano@bs.s.u-tokyo.ac.jp DOI: $10.1508 /$ cytologia.85.3
}

tent stem cells, which supply cells for the differentiation of leaf primordia, similar to the SAM. In this review, we focus on recent studies on the initiation and development of the axillary meristem and on stem cell maintenance during axillary bud formation in two model plants, $A$. thaliana (Arabidopsis) and O. sativa (rice).

\section{Stem cell maintenance in the SAM in Arabidopsis}

The SAM is established during embryogenesis and plays a critical role in post-embryonic development in plants. The SAM has several functional domains, such as the central, peripheral, and rib regions. In the apex of the central region, stem cells are preserved and reproduce by cell division. These stem cells also supply daughter cells to the peripheral region, where the cell fate is determined to differentiate leaf primordia. The balance between stem cell proliferation and cell supply for leaf differentiation is essential to maintain a constant population of stem cells.

This balance, termed stem cell homeostasis, is controlled by the WUSCHEL-CLAVATA (WUS-CLV) negative feedback loop in Arabidopsis (Fig. 1A) (reviewed in Ha et al. 2010, Somssich et al. 2016, Fletcher 2018). The WUS gene, encoding a WOX-type transcription factor, promotes stem cell proliferation and the expression of CLV3. WUS is expressed in the lower part of the central region, called the organizing center, whereas CLV3 
(A)

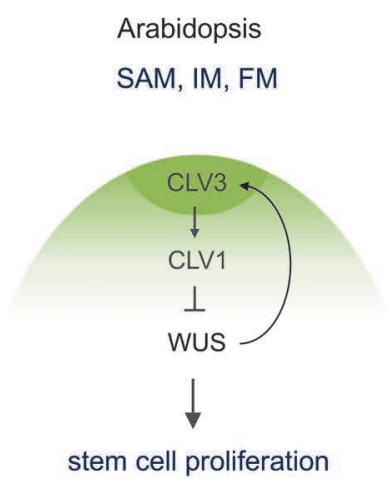

(B)

Rice

SAM

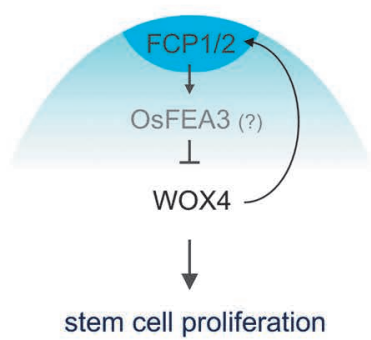

(C)

Rice

FM, IM (SAM)

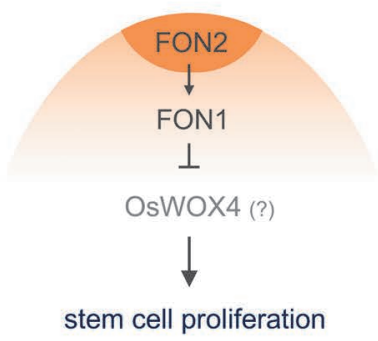

Fig. 1. Schematic representation of the regulation of stem cell maintenance in Arabidopsis (A) and rice (B, C).

is expressed in the stem cell region. Therefore, WUS acts non-cell autonomously to promote stem cell proliferation and $C L V 3$ induction. This non-cell autonomous action of $W U S$ is explained by the movement of WUS protein from the organizing center to the stem cell region (Yadav et al. 2011). An apical-basal gradient of HAIRY MERISTEM (HAM) proteins confines expression of CLV3 to the apical region of the SAM (Zhou et al. 2018). HAMs prevent activation of $C L V 3$ by interacting with WUS in the organizing center where the HAM level is high, whereas WUS induces CLV3 expression in the apical region where the HAM level is low.

CLV3 encodes a small secreted protein with a CLE domain, which is processed into a small peptide of 13 amino acids, called a CLV3-CLE peptide (Kondo et al. 2006). The CLV3-CLE peptide further undergoes chemical modification, including proline hydroxylation and hydroxyproline arabinosylation (Ohyama et al. 2009). The modified CLV3-CLE peptide is perceived by CLV1, an LRR-type receptor kinase, whose expression domain encompasses the organizing center, that is, WUS expression domain (Ogawa et al. 2008). The CLV3 signal ultimately represses WUS expression. Thus, CLV3 negatively regulates stem cell proliferation through the repression of WUS. Accordingly, stem cells are maintained at an almost constant number by this WUS-CLV negative feedback loop.

Stem cell maintenance during axillary meristem development in Arabidopsis

The axillary meristem develops in the leaf axil and, after its establishment, preserves stem cells in the apical region of its central region, similar to the SAM. Leaf primordia are differentiated from the axillary meristem and form axillary buds together with the meristem. After outgrowth of the bud, the axillary meristem acts as the SAM in the secondary shoot (branch).

On the basis of morphological studies, two alternative models have been proposed for the origin of the axillary meristem (Steeves and Sussex 1989). In the 'detached meristem' model, a few pluripotent undifferentiated cells are detached from the primary SAM and preserved in the leaf axil. The axillary meristem develops later from these cells. Alternatively, in the 'de novo induction' model, differentiated cells are re-specified as undifferentiated meristematic cells, which are subsequently used to form the axillary meristem.

The observation that SHOOT MERISTEMLESS (STM), a marker of undifferentiated cells in the SAM, is expressed in the leaf axil before initiation of the axillary meristem supports the former model, but is not sufficient to exclude the latter model (Long and Barton 2000, Greb et al. 2003). Recent studies using live cell imaging have clearly shown that stem cells derived from the SAM are progenitors of the axillary meristems in Arabidopsis and tomato, supporting the 'detached meristem' model (Burian et al. 2016, Shi et al. 2016). In addition, Burian et al. (2016) have shown that the progenitor cells are maintained in a quiescent state after being separated from the stem cell pool in the SAM to minimize mutation by DNA replication. Shi et al. (2016) confirmed the importance of the STM-expressing cells in the leaf axil by a laser ablation experiment, and revealed that upregulation of STM expression by REVOLUTA (REV) is required for initiation of axillary meristem development.

Cytokinin is also necessary to initiate axillary meristem development in Arabidopsis. Cytokinin signal is observed prior to an accumulation of WUS transcripts (Wang et al. 2017). Neither cytokinin signaling nor WUS expression is observed in the leaf axil of mutants defective in axillary bud formation, such as lateral suppressor (las) or rev. Cytokinin signaling is needed for de novo induction of WUS in the leaf axil: some type-B ARABIDOPSIS RESPONSE REGULATORs (ARRs), such as ARR1 and ARR2, directly activate WUS expression (Wang et al. 2017). Consistent with this, no WUS expression is observed in the arrl mutant.

Expression of CLV3 begins slightly but clearly later than that of WUS (Xin et al. 2017) during axillary meristem formation. Given that $W U S$ promotes CLV3 expression in the SAM, the timing of the expression of the two genes suggests that $W U S$ also induces $C L V 3$ expression 
in axillary meristem development. Interestingly, CLV3 is expressed in the central part of the mass of undifferentiated cells that develops into the axillary meristem, and this CLV3-expression region overlaps substantially with the WUS-expression region (Xin et al. 2017, Zhou et al. 2018). Soon after, expression of CLV3 becomes to be localized to the apical region of the mass, similar to its expression in the SAM. This localized CLV3 expression, which is distinct from $W U S$ expression, might indicate near-completion of the axillary meristem. The apicalbasal gradient of HAM also confines CLV3 expression to the apical region of the axillary meristem, similar to its function in the SAM (Zhou et al. 2018). In the clv3 mutant, the expression domain of WUS is expanded in the axillary meristem (Xin et al. 2017). This is similar to the observation that WUS is expressed widely in the SAM of clv3. These observations suggest that stem cells are maintained during axillary meristem development by a genetic mechanism that is similar to the pathway regulating stem cells in the SAM.

\section{Stem cell maintenance in established meristems in rice}

The CLV-like signaling that negatively regulates stem cell proliferation in Arabidopsis is essentially conserved in established meristems such as the SAM and the flower meristem (FM) in rice (Fig. 1B, C) (reviewed in Pautler et al. 2013, Tanaka et al. 2014, Somssich et al. 2016, Fletcher 2018). A mutation in FLORAL ORGAN NUMBER1 (FON1) or FON2 (also called FON4) leads to an enlargement of both the FM and the inflorescence meristem (IM) (Suzaki et al. 2004, 2006, Chu et al. 2006, Suzuki et al. 2019a). FON1 encodes a CLV1-like LRR-type receptor kinase, whereas FON2 encodes a small protein containing the CLE domain, which is highly similar to the CLE domain of CLV3. The FON2 expression domain is expanded in the enlarged FMs and IMs of the fon 1 and fon 2 mutants, suggesting that FON2 marks stem cells in rice, similar to the function of CLV3 in Arabidopsis (Suzaki et al. 2006, Suzuki et al. 2019a). Overexpression of FON2 severely reduces the number of branches, spikelets (flowers), and floral organs, probably due to a decrease in stem cells in the IM and FM (Suzaki et al. 2006). In the fon 1 mutant, FON2 overexpression does not bring about any obvious defects in the flower, suggesting that FON1 act as a receptor for the FON2CLE peptide. These findings indicate that FON signaling negatively regulates stem cell maintenance in rice, similar to CLV signaling in Arabidopsis (Fig. 1A, C).

Unlike Arabidopsis, rice has multiple pathways that negatively regulate stem cell maintenance. FON2 SPARE1 (FOS1) acts redundantly with FON2 in the FM in wild rice species and indica strains, but mainly lacks function in japonica strains due to a mutation at the processing site of the signal peptide (Suzaki et al. 2009). FON2-LIKE CLE PROTEIN1 (FCP1) and FCP2 have been shown to be redundantly involved in negatively regulating main- tenance of the vegetative SAM (Fig. 1B) (Suzaki et al. 2008). Simultaneous downregulation of both FCP1 and $F C P 2$ in seedlings causes expansion of the FON2 expression domain in the SAM and inhibition of leaf differentiation (Ohmori et al. 2013). Overexpression of FCP1 leads to termination of the SAM in regenerated shoots, unlike FON2, whose overexpression has relatively weak effects on shoot regeneration (Suzaki et al. 2008). Because the effect of FCP1 overexpression is also observed in the fon 1 mutant, the FCP1 signal is probably transmitted through a receptor other than FON1. In maize (Zea mays), the FCP1 receptor has been identified as FASCIATED EAR3 (FEA3) (Je et al. 2016). A mutation in either ZmFCP1 or $F E A 3$ results in a marked enlargement of the IM, and both genes function in the same genetic pathway. This FCP1FEA3 pathway has been shown to be also conserved in Arabidopsis (Je et al. 2016).

Unlike the CLV-like genes, the function of the WUS ortholog TILLARS ABSENT1 (TAB1) is not conserved in rice (Tanaka et al. 2015, Suzuki et al. 2019b). In wild type, TAB1 is not expressed in the vegetative SAM. A loss-of-function mutant of TAB1 has been isolated by the TILLING (targeting induced local lesions in genomes) method and shows normal growth of the primary shoot (Tanaka et al. 2015). The tabl mutant has a normal SAM and produces the same number of leaves in the primary shoot as wild type. In addition, the stem cell marker FON2 and undifferentiated cell marker OSH1, an ortho$\log$ of STM, are expressed similarly in the SAM in both tabl and wild-type (Tanaka et al. 2015, Tanaka and Hirano 2020). Collectively, these results indicate that TAB1 does not function in stem cell maintenance in the SAM.

Instead, another WOX gene, OsWOX4, is involved in maintenance of the SAM in rice (Fig. 1B) (Ohmori et al. 2013). OsWOX4 is expressed in the various types of aerial meristem, such as the vegetative SAM, IMs, FMs, and branch meristems (BMs). RNA silencing of OsWOX4 leads to downregulation of FON2 and OSH1 in the vegetative SAM of seedlings, resulting in termination of the SAM. Similar to Arabidopsis WUS, OsWOX4 promotes cytokinin signaling (Gordon et al. 2009, Chickarmane et al. 2012). In addition, OsWOX4 is negatively regulated by FCP1. Therefore, the OsWOX4-FCP1 pathway seems to play a role in stem cell maintenance in the SAM in rice, similar to the WUS-CLV pathway in Arabidopsis (Fig. 1A, B). WOX4 is required for the maintenance of vascular stem cells in Arabidopsis (Hirakawa et al. 2010), suggesting that two orthologous genes have a similar function in stem cell maintenance but have diversified during the evolution rice and Arabidopsis to perform their functions in different meristematic tissues. In rice, $O s W O X 4$ is also important in early leaf development (Yasui et al. 2018). 
(A)

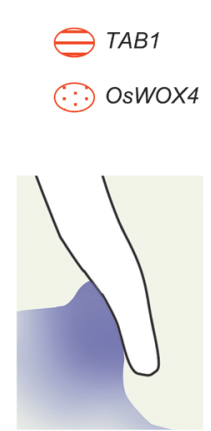

stage 0
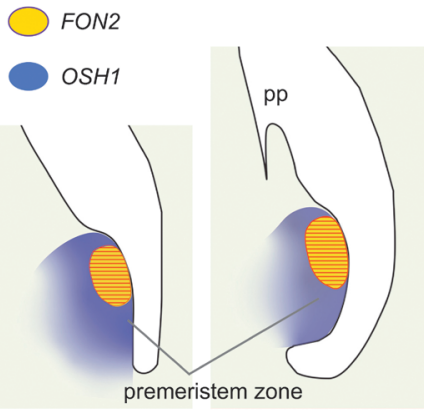

stage 1

stage 2

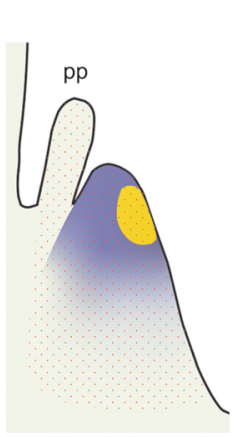

stage 3

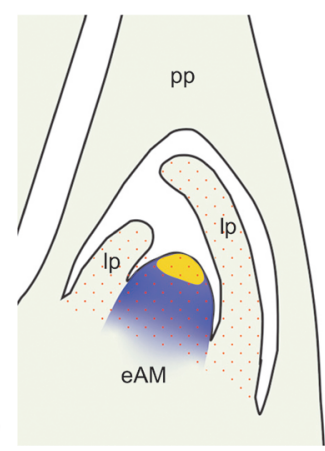

stage 4

(B)
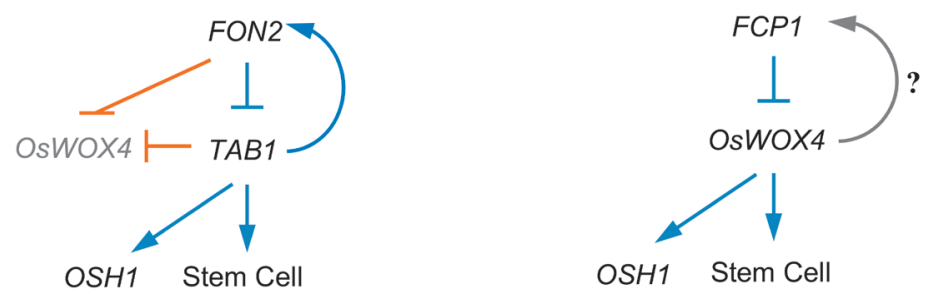

Fig. 2. Genetic regulation of stem cell maintenance during axillary meristem development in rice. Upper panel, the four stages of axillary meristem development. Lower panel, the two pathways involved in regulation of stem cell maintenance. eAM, established axillary meristem; lp, leaf primordia; pp, prophyll.

Stem cell maintenance during axillary meristem development in rice

Although not required for stem cell maintenance in the SAM, TAB1 is essential for the formation of axillary shoots (called tillers) in rice (Tanaka et al. 2015). The requirement of the $W U S$ ortholog (also called MONOCULM1 and STERILE AND REDUCED TILLERING1) for tiller formation is supported by the studies on gene cloning using reduced tiller mutants (Lu et al. 2015, Mjomba et al. 2016).

Formation of the axillary bud is compromised in the tab1/moc3 mutant (Lu et al. 2015, Tanaka et al. 2015). In wild type, the axillary bud is formed on the culm (stem) near the boundary region between the culm and the leaf primordium. In the tabl mutant, the progress of axillary bud formation is arrested at various developmental stages due to a failure of axillary meristem development (Tanaka et al. 2015). In wild-type rice, a dome-like axillary meristem (similar to the shape of the SAM) is formed via a premeristem zone, where a transient meristem differentiates the primordium of the prophyll, a special leaf enclosing the axillary bud. TABI is expressed in this premeristem zone (Fig. 2A). Whereas OSH1 is expressed in the premeristem zone and in subsequent developing meristems in wild type (stage 1 and stage 2), its expression is strongly reduced or absent in tab1. Therefore, TAB1 seems to maintain cells in an undifferentiated state in the premeristem zone. Interestingly, TAB1 expression is transient and disappears at the later stages of axillary meristem development (stage 3 and stage 4). At the same time, WOX4 starts to be expressed instead of TABI (Fig. 2A). Thus, two related $W O X$ genes, $T A B 1$ and $W O X 4$, sequentially promote development of the axillary meristem in rice (Fig. 2B). OSH1 is also plays an essential role in axillary meristem development, because axillary bud formation is profoundly affected in the osh1 mutant (Tanaka et al. 2019).

Expression of the stem cell marker FON2 is observed at the initial stages of axillary meristem development at almost the same time as the appearance of the bulge of the premeristem zone (Fig. 2A, stage 1) (Tanaka and Hirano 2020). This suggests that stem cell identity is established very early in development of the axillary meristem. FON2 is expressed at the apical region of the premeristem zone from stage 1; this early localization of FON2 at the apical region contrasts with the localization of Arabidopsis CLV3, which alters its expression pattern during the course of axillary meristem development (Xin et al. 2017, Zhou et al. 2018). Expression of FON2 is not observed in tab1, suggesting that TAB1 induces FON2 and that stem cells are not maintained in the absence of TAB1 (Tanaka and Hirano 2020). MOC1 and LAX PANICE1 (LAX1), which are required for axillary bud formation, are expressed normally in $t a b 1$, suggesting that $T A B 1$ functions downstream of these two genes (Komatsu et al. 2003, Li et al. 2003, Tanaka and Hirano 2020). In addition, axillary meristem development is initiated in tabl, although developmental arrest occurs soon afterwards. Overall, the absence of FON2 expression in $t a b 1$ strongly suggests that $T A B 1$ is required to maintain, but not initiate, stem cells during axillary meristem development.

In the fon 2 mutant, the size of the premeristem zone is slightly enlarged and the expression domain of FON2 is expanded as compared with wild type. Therefore, it seems likely that stem cell proliferation is negatively 
regulated by FON2. Consistent with this idea, overexpression of FON2 partially inhibits meristem maintenance during axillary meristem development, resulting in a reduction in tiller number (Tanaka and Hirano 2020). TAB1 expression is elevated in fon2. Accordingly, the genetic relationship between TAB1 and FON2 in axillary meristem development in rice is highly similar to that of WUS and CLV3 in the SAM in Arabidopsis (Figs. 1A, 2B).

The tab1 fon 2 double mutant generates tillers to some extent, suggesting that the fon 2 mutation partially suppress the tabl phenotype (Tanaka and Hirano 2020). This genetic interaction between TAB1 and FON2 is in high contrast to that between WUS and CLV3 in Arabidopsis, where the wus clv3 double mutant exhibits the same phenotype as the wus single mutant, indicating that wus is completely epistatic to clv3 (Laux et al. 1996). The enigmatic phenotype of the tab1 fon 2 mutant can be explained by the observation that WOX4 is expressed precociously in the premeristem zone in the absence of FON2 (Tanaka and Hirano 2020). Because the induction of WOX4 by the TAB1 promoter in tabl rescues its mutant phenotype, precociously expressed WOX4 seems to function in place of TAB1 during axillary bud formation in the tab1 fon 2 double mutant.

In summary, WOX4 promotes stem cells in the SAM, whereas $T A B 1$ promotes them during axillary meristem development. Similarly, FCP1 negatively regulates stem cell proliferation in the SAM, whereas FON2 negatively regulates it during axillary bud formation. Thus, a similar genetic mechanism regulates stem cell maintenance in both the established meristem and the developing meristem during axillary bud formation. A similar mechanism involving WUS and CLV3 functions in SAM maintenance and in axillary meristem development in Arabidopsis, as described above. In rice, however, the involvement of WOX4 seems to make the mechanism underlying stem cell maintenance more complex as compared with Arabidopsis.

\section{Future perspective}

Recent studies demonstrate that the fundamental mechanism underlying stem cell maintenance is conserved in Arabidopsis and rice, two relatively distant species among angiosperms. However, the specific genes and their contribution differ between the two species: for example, the function of WUS to regulate stem cell maintenance in both the SAM and developing meristems in Arabidopsis has been divided between two genes, TAB1 and $W O X 4$, in rice. In legumes such as Lotus japonicus, the gene that is orthologous to Arabidopsis CLV1 and rice FON1 does not have a role in stem cell maintenance, but regulates the number of nodules (Nishimura et al. 2002). Therefore, further studies using more varied species are needed to understand the conserved mechanisms underlying stem cell maintenance among angiosperms and the mechanisms specific to each species.

\section{Acknowledgements}

This study was supported by MEXT/JSPS KAKENHI (17H03745 to H.-Y. H.; 19K16160 to W. T.).

\section{References}

Barbier, F. F., Dun, E. A., Kerr, S. C., Chabikwa, T. G. and Beveridge, C. A. 2019. An update on the signals controlling shoot branching. Trends Plant Sci. 24: 220-236.

Bell, A. D. 2008. Plant Form: An Illustrated Guide to Flowering Plant Morphology. Timber Press, Portland.

Burian, A., Barbier de Reuille, P. and Kuhlemeier, C. 2016. Patterns of stem cell divisions contribute to plant longevity. Curr. Biol. 26: 1385-1394.

Chickarmane, V. S., Gordon, S. P., Tarr, P. T., Heisler, M. G. and Meyerowitz, E. M. 2012. Cytokinin signaling as a positional cue for patterning the apical-basal axis of the growing Arabidopsis shoot meristem. Proc. Natl. Acad. Sci. U.S.A. 109: 4002-4007.

Chu, H., Qian, Q., Liang, W., Yin, C., Tan, H., Yao, X., Yuan, Z., Yang, J., Huang, H., Luo, D., Ma, H. and Zhang, D. 2006. The FLORAL ORGAN NUMBER4 gene encoding a putative ortholog of Arabidopsis CLAVATA3 regulates apical meristem size in rice. Plant Physiol. 142: 1039-1052.

Dhaliwal, A., Dhaliwal, R. S., Kaur, N. and Gupta, R. C. 2018. Cytomorphological study in genus Cenchrus L.: An important medicinal plant from north India (family: Poaceae). Cytologia 83: $45-52$.

Domagalska, M. A. and Leyser, O. 2011. Signal integration in the control of shoot branching. Nat. Rev. Mol. Cell Biol. 12: 211-221.

Fletcher, J. C. 2018. The CLV-WUS stem cell signaling pathway: A roadmap to crop yield optimization. Plants 7: E87.

Gordon, S. P., Chickarmane, V. S., Ohno, C. and Meyerowitz, E. M. 2009. Multiple feedback loops through cytokinin signaling control stem cell number within the Arabidopsis shoot meristem. Proc. Natl. Acad. Sci. U.S.A. 106: 16529-16534.

Greb, T., Clarenz, O., Schafer, E., Muller, D., Herrero, R., Schmitz, G. and Theres, K. 2003. Molecular analysis of the LATERAL SUPPRESSOR gene in Arabidopsis reveals a conserved control mechanism for axillary meristem formation. Genes Dev. 17: 1175-1187.

Ha, C. M., Jun, J. H. and Fletcher, J. C. 2010. Shoot apical meristem form and function. Curr. Top. Dev. Biol. 91: 103-140.

Hirakawa, Y., Kondo, Y. and Fukuda, H. 2010. TDIF peptide signaling regulates vascular stem cell proliferation via the WOX4 homeobox gene in Arabidopsis. Plant Cell 22: 2618-2629.

Je, B. I., Gruel, J., Lee, Y. K., Bommert, P., Arevalo, E. D., Eveland, A. L., Wu, Q., Goldshmidt, A., Meeley, R., Bartlett, M., Komatsu, M., Sakai, H., Jonsson, H. and Jackson, D. 2016. Signaling from maize organ primordia via FASCIATED EAR3 regulates stem cell proliferation and yield traits. Nat. Genet. 48: 785-791.

Komatsu, K., Maekawa, M., Ujiie, S., Satake, Y., Furutani, I., Okamoto, H., Shimamoto, K. and Kyozuka, J. 2003. LAX and SPA: Major regulators of shoot branching in rice. Proc. Natl. Acad. Sci. U.S.A. 100: 11765-11770.

Kondo, T., Sawa, S., Kinoshita, A., Mizuno, S., Kakimoto, T., Fukuda, H. and Sakagami, Y. 2006. A plant peptide encoded by CLV3 identified by in situ MALDI-TOF MS analysis. Science 313: 845-848.

Kumar, A., Meena, H. S., Ram, B., Priyamedha., Sharma, A., Yadav, S., Singh, V. V. and Rai, P. K. 2018. Some cytomorphological evidence for synthesis of interspecific hybrids between Brassica 
juncea and autotetraploid B. fruticulosa. Cytologia 83: 421-426.

Laux, T., Mayer, K. F. X., Berger, J. and Jürgens, G. 1996. The WUSCHEL gene is required for shoot and floral meristem integrity in Arabidopsis. Development 122: 87-96.

Li, X., Qian, Q., Fu, Z., Wang, Y., Xiong, G., Zeng, D., Wang, X., Liu, X., Teng, S., Hiroshi, F., Yuan, M., Luo, D., Han, B. and Li, J. 2003. Control of tillering in rice. Nature 422: 618-621.

Long, J. and Barton, M. K. 2000. Initiation of axillary and floral meristems in Arabidopsis. Dev. Biol. 218: 341-353.

Lu, Z., Shao, G., Xiong, J., Jiao, Y., Wang, J., Liu, G., Meng, X., Liang, Y., Xiong, G., Wang, Y. and Li, J. 2015. MONOCULM 3, an ortholog of WUSCHEL in rice, is required for tiller bud formation. J. Genet. Genomics 42: 71-78.

Mjomba, F. M., Zheng, Y., Liu, H., Tang, W., Hong, Z., Wang, F. and $\mathrm{Wu}, \mathrm{W} .22016$. Homeobox is pivotal for OsWUS controlling tiller development and female fertility in rice. G3 6: 2013-2021.

Nakayama, H., Yamaguchi, T. and Tsukaya, H. 2012. Acquisition and diversification of cladodes: Leaf-like organs in the genus Asparagus. Plant Cell 24: 929-940.

Nasirvand, S., Zakaria, R. A., Zare, N. and Esmaeilpoor, B. 2018. Polyploidy induction in parsley (Petroselinum crispum L.) by colchicine treatment. Cytologia 83: 393-396.

Nishimura, R., Hayashi, M., Wu, G. J., Kouchi, H., ImaizumiAnraku, H., Murakami, Y., Kawasaki, S., Akao, S., Ohmori, M., Nagasawa, M., Harada, K. and Kawaguchi, M. 2002. HAR1 mediates systemic regulation of symbiotic organ development. Nature 420: 426-429.

Ogawa, M., Shinohara, H., Sakagami, Y. and Matsubayashi, Y. 2008. Arabidopsis CLV3 peptide directly binds CLV1 ectodomain. Science 319: 294

Ohmori, Y., Tanaka, W., Kojima, M., Sakakibara, H. and Hirano, H. Y. 2013. WUSCHEL-RELATED HOMEOBOX4 is involved in meristem maintenance and is negatively regulated by the CLE gene FCP1 in rice. Plant Cell 25: 229-241.

Ohyama, K., Shinohara, H., Ogawa-Ohnishi, M. and Matsubayashi, Y. 2009. A glycopeptide regulating stem cell fate in Arabidopsis thaliana. Nat. Chem. Biol. 5: 578-580.

Pautler, M., Tanaka, W., Hirano, H. Y. and Jackson, D. 2013. Grass meristems I: Shoot apical meristem maintenance, axillary meristem determinacy and the floral transition. Plant Cell Physiol. 54: $302-312$.

Shi, B., Zhang, C., Tian, C., Wang, J., Wang, Q., Xu, T., Xu, Y., Ohno, C., Sablowski, R., Heisler, M. G., Theres, K., Wang, Y. and Jiao, Y. 2016. Two-step regulation of a meristematic cell population acting in shoot branching in Arabidopsis. PLoS Genet. 12: e1006168.

Shiogai, S., Tamotsu, S. and Sakai, A. 2018. $\mathrm{C}_{3}$-like photosynthetic properties of senescing maize leaves are accompanied by preferential senescence of mesophyll cells. Cytologia 83: 387-391.

Soltis, P. S. and Soltis, D. E. 2014. Flower Diversity and Angiosperm Diversification. In: Fornara, F. (ed.). The Molecular Genetics of Floral Transition and Flower Development. Elsevier, Amsterdam. pp. 221-262.

Somssich, M., Je, B. I., Simon, R. and Jackson, D. 2016. CLAVATAWUSCHEL signaling in the shoot meristem. Development 143: 3238-3248.

Steeves, T. A. and Sussex, I. M. 1989. Patterns in Plant Development. Cambridge University Press, Cambridge, UK.

Suzaki, T., Ohneda, M., Toriba, T., Yoshida, A. and Hirano, H.-Y. 2009. FON2 SPARE1 redundantly regulates floral meristem maintenance with FLORAL ORGAN NUMBER2 in rice. PLoS Genet. 5: e1000693.

Suzaki, T., Sato, M., Ashikari, M., Miyoshi, M., Nagato, Y. and Hirano, H. Y. 2004. The gene FLORAL ORGAN NUMBER1 regulates floral meristem size in rice and encodes a leucine-rich repeat receptor kinase orthologous to Arabidopsis CLAVATA1. Development 131: 5649-5657.

Suzaki, T., Toriba, T., Fujimoto, M., Tsutsumi, N., Kitano, H. and Hirano, H. Y. 2006. Conservation and diversification of meristem maintenance mechanism in Oryza sativa: Function of the FLORAL ORGAN NUMBER2 gene. Plant Cell Physiol. 47: 1591-1602.

Suzaki, T., Yoshida, A. and Hirano, H.-Y. 2008. Functional diversification of CLAVATA3-related CLE proteins in meristem maintenance in rice. Plant Cell 20: 2049-2058.

Suzuki, C., Tanaka, W. and Hirano, H. Y. 2019a. Transcriptional corepressor ASP1 and CLV-like signaling regulate meristem maintenance in rice. Plant Physiol. 180: 1520-1534.

Suzuki, C., Tanaka, W., Tsuji, H. and Hirano, H. Y. 2019b. TILLERS $A B S E N T 1$, the WUSCHEL ortholog, is not involved in stem cell maintenance in the shoot apical meristem in rice. Plant Signal. Behav. 14: e1640565.

Tanaka, W. and Hirano, H. Y. 2020. Antagonistic action of TILLERS ABSENT1 and FLORAL ORGAN NUMBER2 regulates stem cell maintenance during axillary meristem development in rice. New Phytol. 225: 974-984.

Tanaka, W., Ohmori, Y., Ushijima, T., Matsusaka, H., Matsushita, T., Kumamaru, T., Kawano, S. and Hirano, H.-Y. 2015. Axillary meristem formation in rice requires the WUSCHEL ortholog TILLERS ABSENT1. Plant Cell 27: 1173-1184.

Tanaka, W., Toriba, T. and Hirano, H.-Y. 2014. Flower Development in Rice. In: Fornara, F. (ed.). The Molecular Genetics of Floral Transition and Flower Development. Elsevier, Amsterdam. pp. 221-262.

Tanaka, W., Tshuda, K. and Hirano, H.-Y. 2019. Class I KNOX gene OSH1 is indispensable for axillary meristem development in rice. Cytologia 84: 343-346.

Tsukaya, H. 2018. Leaf shape diversity with an emphasis on leaf contour variation, developmental background, and adaptation. Semin. Cell Dev. Biol. 79: 48-57.

Wang, B., Smith, S. M. and Li, J. 2018. Genetic regulation of shoot architecture. Annu. Rev. Plant Biol. 69: 437-468.

Wang, J., Tian, C., Zhang, C., Shi, B., Cao, X., Zhang, T. Q., Zhao, Z., Wang, J. W. and Jiao, Y. 2017. Cytokinin signaling activates WUSCHEL expression during axillary meristem initiation. Plant Cell 29: 1373-1387.

Wang, Q., Hasson, A., Rossmann, S. and Theres, K. 2016. Divide et impera: Boundaries shape the plant body and initiate new meristems. New Phytol. 209: 485-498.

Xin, W., Wang, Z., Liang, Y., Wang, Y. and Hu, Y. 2017. Dynamic expression reveals a two-step patterning of WUS and CLV3 during axillary shoot meristem formation in Arabidopsis. J. Plant Physiol. 214: 1-6.

Yadav, R. K., Perales, M., Gruel, J., Girke, T., Jönsson, H. and Reddy, G. V. 2011. WUSCHEL protein movement mediates stem cell homeostasis in the Arabidopsis shoot apex. Genes Dev. 25: 2025-2030.

Yamaguchi, T., Yano, S. and Tsukaya, H. 2010. Genetic framework for flattened leaf blade formation in unifacial leaves of Juncus prismatocarpus. Plant Cell 22: 2141-2155.

Yasui, Y., Ohmori, Y., Takebayashi, Y., Sakakibara, H. and Hirano, H.-Y. 2018. WUSCHEL-RELATED HOMEOBOX4 acts as a key regulator in early leaf development in rice. PLoS Genet. 14: e1007365.

Zhou, Y., Yan, A., Han, H., Li, T., Geng, Y., Liu, X. and Meyerowitz, E. M. 2018. HAIRY MERISTEM with WUSCHEL confines CLAVATA3 expression to the outer apical meristem layers. Science 361: 502-506. 